

\title{
Modelación econométrica de la eco-eficiencia en las empresas eléctricas de Costa Rica utilizando smart grid
}

\author{
Rodrigo Espinosa Porras \\ respinoza90153@ufide.ac.cr, Universidad Fidélitas
}

\begin{abstract}
Eco-efficiency expresses the efficiency with which ecological resources are used to meet human needs. It is considered as the average of an output divided by an input; the first is the value of products and services produced by a company, sector, or economy as a whole, and the second, the sum of the environmental pressures generated by the company, sector, or economy. Eco-efficiency is measured depending on the typification of indicators of inputs and outputs. For this, an econometric modeling was established, based on Harrod-Domar's macroeconomic theory, which expresses the indispensable conditions in a capitalist or market economy, to generate the volume of necessary global demand and, thus, to allow the sustained and balanced development, without abandoning the Keynesian system. This model considers the productive capacity as a variable over time and establishes that an investment differential is equal to the savings differential. In this article, we study the behavior of the HarrodDomar model to determine a basic econometric equation that provides an improvement in the eco-efficiency curve applied to Costa Rican electricity companies. The improvement curve in environmental technology aims to demonstrate theoretically that there is economic growth, by obtaining a reduction in the percentage of expenses or outputs against the environment, with the implementation of smart grid technologies.
\end{abstract}

Keywords: Smart Grid, Econometry, Electrical Company, Ecoeficiency, Growth.

\section{Resumen}

La eco-eficiencia expresa la eficiencia con la cual los recursos ecológicos se utilizan para satisfacer las necesidades humanas. Se considera como el promedio de un output dividido por un input; el primero es el valor de los productos y servicios producidos por una empresa, sector, o economía en su conjunto, y el segundo, la suma de las presiones ambientales generadas por la empresa, sector, o economía. La eco-eficiencia se mide dependiendo de la tipificación de indicadores de inputs y outputs, para ello se estableció una modelación econométrica, basada en la teoría macroeconómica de Harrod-Domar, que expresa las condiciones indispensables en una economía capitalista o de mercado, para generar el volumen de demanda global necesario y, así, permitir el desarrollo sostenido y equilibrado, sin abandonar el sistema keynesiano. Este modelo considera la capacidad productiva como una variable a lo largo del tiempo y establece que un diferencial de inversión es igual al diferencial del ahorro. En este artículo, se estudia el comportamiento del modelo de Harrod-Domar para determinar una ecuación econométrica básica que, proporcione una mejora en la curva de eco-eficiencia aplicada a las empresas eléctricas de Costa Rica. La curva de la mejora en tecnología ambiental pretende demostrar teóricamente que existe un crecimiento económico, al obtener una disminución del porcentaje de gastos u outputs en contra del ambiente, con la implementación de tecnologías de smart grid.

Palabras clave: SMART GRID, Econometría, Empresa Eléctrica, Eco-Eficiencia, Crecimiento.

\section{Introducción}

La presente investigación metodológica se fundamenta en plantear un modelo económico básico, que sirva como fundamento econométrico o estadístico de la teoría, para desarrollar en una futura tesis doctoral, una respuesta a la incógnita sobre si las empresas eléctricas en Costa Rica están en condiciones de aprovechar y adaptar los cambios tecnológicos mundiales, para mantener y aumentar la ecoeficiencia en la matriz eléctrica nacional, el desarrollo sostenible, ofrecer precios de la electricidad que sean lo más competitivos posibles en el contexto nacional, por medio de una futura integración a una red inteligente o smart grid y demostrar crecimiento económico.

El crecimiento de la demanda energética es el elemento central que impulsa las necesidades de aumento de la oferta y capacidad del sistema eléctrico en general, ese incremento en el consumo, originará en el futuro, impactos 
ambientales, sociales y económicos, sobre los cuales se requiere tomar acciones políticas a fin de minimizarlos (Ministerio de Ambiente, Energía y Telecomunicaciones, 2011).

Una de las oportunidades más importantes que tiene el país, consiste en aprovechar todas las posibilidades de reducción en el crecimiento de la demanda, para que no afecten otras metas de desarrollo y que reduzca la necesidad de instalación de nuevas plantas generadoras de electricidad y el consumo de combustibles. Lo deseable es renovar la demanda de energía del país mediante una matriz energética que asegure el suministro óptimo y continuo de electricidad y combustible, promoviendo el uso eficiente de energía para mantener y mejorar la competitividad del país con predominio de fuentes renovables y al menor costo (Ministerio de Ambiente, Energía y Telecomunicaciones, 2011).

Con un sistema tecnológico de alto impacto como lo es una red inteligente, se puede controlar completamente el consumo y generación de la energía, con lo que se logrará asegurar el abastecimiento y uso en la cantidad, calidad y con diversidad de fuentes, compatibles con el desarrollo sostenible del país; ya que abarca tanto a la empresa eléctrica como a la comunidad en donde se aplique.

Una smart grid de define como el mejorar la eficiencia, la sostenibilidad, la economía y la resiliencia de las redes eléctricas mediante el desarrollo y la demostración de avances en la ciencia de la medición, para mejorar la interoperabilidad de la red y facilitar el uso de la red de distribución como una plataforma habilitadora para los servicios energéticos modernos.

Es por ello que se debe de realizar un análisis de la economía ambiental desde el punto de vista de tecnología, con el fin de determinar una metodología económica, que podrían aplicar las empresas eléctricas en Costa Rica para poder sustentar el desarrollo sostenible y la viabilidad de proyectos de un elevado valor económico, por lo que la propuesta es utilizar el análisis desde la perspectiva de la economía ambiental denominada eco-eficiencia (Lababdeira, León, \& Vázquez, 2007).

La eco-eficiencia en temas tecnológicos, se basa en el modelo económico de crecimiento equilibrado con interés compuesto o exponencial, construidos por Sir Roy Harrod en Inglaterra, y por el profesor E. Domar en Estados Unidos, este modelo tiene dos conceptos básicos: la tasa natural de crecimiento a largo plazo y la llamada tasa garantizada de crecimiento (Lababdeira, León, \& Vázquez, 2007).

Desde el punto de vista de las empresas eléctricas, la metodología econométrica basada en eco-eficiencia demostraría que la curva de la mejora en tecnología ambiental aumenta utilizando smart grid, suponiendo que la mejora es proporcional en la curva de eco-eficiencia $\mathrm{y}$ que hay un crecimiento económico al obtener una disminución del porcentaje de gastos u outputs en contra del ambiente, y por ende un efecto colateral en la reducción de tarifas a nivel país y en el sector comercial un aumento en la competitividad.

Así, esta investigación tiene como objetivo proponer la base hipotética de un modelo econométrico basado en la teoría económica de Harrod-Domar de eco-eficiencia, para determinar si las empresas eléctricas de Costa Rica están en capacidad económica de demostrar crecimiento utilizando la tecnología del smart grid y así contribuir con el desarrollo sostenible, la competitividad en la industria, coadyuvar con la matriz energética nacional, reducir tarifas y obtener beneficios económicos.

\section{Referentes teóricos}

El modelo Harrod-Domar expresa las condiciones que debe tener una economía capitalista o de mercado para generar el volumen de demanda global necesario para permitir el desarrollo sostenido y equilibrado; sin abandonar nunca el sistema keynesiano, este modelo considera la capacidad productiva como una variable a lo largo del tiempo.

Para garantizar el equilibrio a largo plazo no basta con el volumen de ahorro, sino que es preciso que en todo momento se dé una total utilización de la capacidad productiva, incrementada a través de las nuevas inversiones (Gandolfo, 1976).

El modelo de Harrod y Domar pertenece al grupo de modelos basados en el multiplicador y el acelerador. Tiene una gran influencia keynesiana y, como los de Kalecki, Samuelson, Kaldor, Hicks, es tan simplificado que su verificación econométrica parece ser una cuestión bastante difícil (Franco González \& Ramírez Hassan, 2005).

Considerando al tiempo como una variable discreta, el modelo de Harrod-Domar acostumbra a formularse mediante las tres siguientes ecuaciones:

$$
\begin{gathered}
\mathrm{A}_{\mathrm{t}}=\mathrm{s} \mathrm{Y}_{\mathrm{t}} \\
\mathrm{It}=\mathrm{v}\left(\mathrm{Y}_{\mathrm{t}}-\mathrm{Y}_{-}\left(\mathrm{t}_{\mathrm{t}-1}\right)\right) \\
\left(\mathrm{A}_{\mathrm{t}}=\mathrm{I}_{\mathrm{t}}\right)
\end{gathered}
$$

En donde $A$ representa el ahorro, $I$ la inversión e $Y$ la renta.

a. La primera ecuación, es la función de ahorro y expresa el comportamiento de los ahorradores, suponiendo que la cantidad ahorrada es una parte proporcional de la renta. El coeficiente s expresa la propensión media y marginal al ahorro (Franco González \& Ramírez Hassan, 2005).

b. La segunda ecuación define el comportamiento de los empresarios en cuanto a su actitud para invertir. Esta 
actitud se supone de manera que la inversión realizada en el período $t$ es proporcional al incremento de la renta de dicho período con respecto a la del anterior (Franco González \& Ramírez Hassan, 2005).

c. Por último, la tercera ecuación es una de las conocidas relaciones contables, la Inversión es igual al Ahorro en un periodo de tiempo $t$ (Franco González \& Ramírez Hassan, 2005).

Desde un punto de vista económico, las hipótesis de Harrod es esencialmente diferente a las de Domar.

En el de Harrod, aparece una clara hipótesis de comportamiento referente a las decisiones de inversión de los empresarios que da lugar a una función de inversión (Franco González \& Ramírez Hassan, 2005):

- El ahorro depende del ingreso.

- La inversión depende del ingreso.

- En consecuencia, todo ahorro se invierte.

En el modelo de Domar por el contrario, no hay función de inversión (en el sentido de relación de comportamiento), pues su problema es diferente: es determinar la tasa a la cual la inversión debe crecer para que no haya capacidad ociosa, y no introduce función de inversión alguna para explicar cómo ésta puede en realidad crecer a esa tasa (Domar, 1946):

- La inversión incrementa la capacidad productiva.

- La capacidad productiva incrementa a su vez la producción potencial.

- Dicha producción debe ser absorbida por un incremento de la demanda agregada (consumo más inversión).

\section{Conjunción de los modelos}

A pesar de sus diferentes enfoques, ambos modelos se unen para determinar una teoría que explica al final, que, realizando un ahorro, se pude invertir y por ende hay crecimiento.

\section{Modelo Domar:}

El inicio del modelo establece que la tasa natural de crecimiento $\mathrm{g}$ es igual a la relación directa del diferencial de salida $\Delta Y$ (renta), respecto a la inversión agregada e inversamente proporcional a dicha inversión I (Domar, 1946):

$$
\mathrm{g}=\frac{\Delta \mathrm{Y}}{\mathrm{I}}
$$

Debido a que la inversión se debe enfocar en una ganancia comparada con un rédito, Domar propone una ecuación sobre la tasa de ahorro s en la cual relaciona el diferencial del ahorro $\triangle A$ y la salida respecto a la inversión $\Delta Y$ (Domar, 1946).

$$
\mathrm{s}=\frac{\Delta \mathrm{A}}{\Delta \mathrm{Y}}
$$

Ahora, se aplica la relación de la inversión, que establece la diferencia en la inversión $\Delta I$, entre la inversión I total aportada, es igual a multiplicar la tasa diferencial del ahorro $g$ por la tasa natural de crecimiento $s$ (Domar, 1946) :

$$
\frac{\Delta \mathrm{I}}{\mathrm{I}}=\mathrm{g} * \mathrm{~s}
$$

Al sustituir las ecuaciones 4,5 y 6 se tiene la siguiente igualdad:

$$
\frac{\Delta \mathrm{I}}{\mathrm{I}}=\frac{\Delta \mathrm{A}}{\Delta \mathrm{Y}} * \frac{\Delta \mathrm{Y}}{\mathrm{I}}
$$

De la anterior, se desprende que la inversión es igual a la multiplicación de la tasa natural de crecimiento por el ahorro, por lo que al cancelar el diferencial de salida $\Delta Y$, se tiene solo el diferencial de ahorro entre la inversión (Domar, 1946):

$$
\frac{\Delta \mathrm{I}}{\mathrm{I}}=\frac{\Delta \mathrm{A}}{\mathrm{I}}
$$

Al igualar la inversión en ambos lados de la ecuación, obtenemos lo que Domar establece como su máxima económica: un diferencial de inversión es igual al diferencial del ahorro (Domar, 1946).

$$
\Delta \mathrm{I}=\Delta \mathrm{A}
$$

\section{Modelo Harrod:}

Por otro lado, tenemos al modelo de Harrod, el cual establece como un inicio de la ecuación, que el crecimiento $G$ es la igualdad del diferencial de salida $\Delta Y$ entre la salida Y (Franco González \& Ramírez Hassan, 2005).

$$
\mathrm{G}=\frac{\Delta \mathrm{Y}}{\mathrm{Y}}
$$


La siguiente relación que se plantea, es que la inversión neta $C$ es igual a la inversión $I$ sobre el diferencial de salida $\Delta Y$ (Franco González \& Ramírez Hassan, 2005).

$$
\mathrm{C}=\frac{\mathrm{I}}{\Delta \mathrm{Y}}
$$

$\mathrm{Al}$ igual que Domar, Harrod expone que la tasa de ahorro $\mathrm{s}$ relaciona el diferencial del ahorro $A$ y la salida respecto a la inversión $Y$ (Franco González \& Ramírez Hassan, 2005).

$$
\mathrm{s}=\frac{\mathrm{A}}{\mathrm{Y}}
$$

Con ello se iguala la ecuación y se muestra que la tasa de ahorro s es la multiplicación del crecimiento $G$ por la inversión neta $C$ (Franco González \& Ramírez Hassan, 2005).

$$
\mathrm{G} * \mathrm{C}=\mathrm{s}
$$

Al sustituir las ecuaciones 10,11 y 12 con respecto a la inversión, ahorro y salida, se tiene la siguiente ecuación (Franco González \& Ramírez Hassan, 2005):

$$
\frac{\Delta \mathrm{Y}}{\mathrm{Y}} * \frac{\mathrm{I}}{\Delta \mathrm{Y}}=\frac{\mathrm{A}}{\mathrm{Y}}
$$

Al despejar y cancelar las variables de diferencial de salida $\Delta Y$, e igualar la salida $Y$, se tiene el mismo resultado que Domar, concluyendo que la inversión es igual al ahorro (Franco González \& Ramírez Hassan, 2005)

$$
\begin{aligned}
\frac{I}{Y} & =\frac{A}{Y} \\
I & =A
\end{aligned}
$$

Ahora bien, si se sustituye la tasa de ahorro en la ecuación 15 , se obtiene que la inversión entre su entrada $Y$, es igual a dicha tasa $\mathrm{s}$.

$$
\frac{\mathrm{I}}{\mathrm{Y}}=\mathrm{s}
$$

O bien:

$$
\mathrm{A}=\mathrm{I}=\mathrm{s} * \mathrm{Y}
$$

En este punto ambas teorías se unifican: establece para Harrod que el ciclo económico es una parte integrada del camino del crecimiento y para Domar, no lo es, pero se acomoda en su modelo al permitir que $\sigma$ (productividad media de la inversión) fluctúe (Labandeira, Leon, \& Vásquez, 2007).

Harrod y Domar han empleado los mismos coeficientes de aceleración, pero con símbolos diferentes. El coeficiente de aceleración de Harrod se llama psicológico, el de Domar es la tecnología, para ello, se supone que al aplicar tecnología como la de un smart grid en las empresas eléctricas en Costa Rica, se podrían obtener beneficios por el crecimiento económico, si utilizamos la ecuación de crecimiento clásico, podemos inferir la siguiente ecuación (European Communities, 2002):

$$
\Delta \mathrm{Y}=\propto * \mathrm{I}
$$

Donde; $\alpha$ es la tasa de crecimiento del output respecto al capital invertido e $I$ es la inversión agregada que depende del ahorro agregado, por lo que se tiene:

$$
\mathrm{I}=\mathrm{s} *(1-\mathrm{e}) * \mathrm{Y}(20)
$$

Por otra parte, $\mathrm{s}$ es la tasa de ahorro respecto a la renta real, teniendo en cuenta los gastos ambientales y suponiendo que es una fracción de la renta real total, e (European Communities, 2002).

Al relacionar las dos ecuaciones, se obtiene el modelo Harrod-Domar modificado del crecimiento en el output real (European Communities, 2002):

$$
\frac{\Delta \mathrm{Y}}{\mathrm{Y}}=\propto \mathrm{s}(1-\mathrm{e})
$$

Ahora se supone, que el crecimiento en eco-eficiencia $P$, está determinado por la función (European Communities, 2002);

$$
\frac{\Delta \mathrm{P}}{\mathrm{P}}+\pi+\mathrm{F}(\mathrm{e})
$$

Donde $\pi$ indica la tendencia a largo plazo de las mejoras en eco-eficiencia y $F(e)$ es una función cóncava de la renta real, $e$.

La teoría de esta ecuación dice que para mantener la tasa de crecimiento de eco-eficiencia por encima de la tendencia $\pi$, 
se requiere que un porcentaje creciente de output se destine a gastos ambientales, o que en otras palabras se podría decir es una compensación por medio de utilización de dinero, en la Gráfica \#1, se muestra el modelo de crecimiento con gastos ambientales, en donde la curva de eco-eficiencia es mejorada con tecnología ambiental, y por ende un aumento en el crecimiento económico (European Communities, 2002).

Gráfico 1. Modelo de crecimiento con gastos ambientales

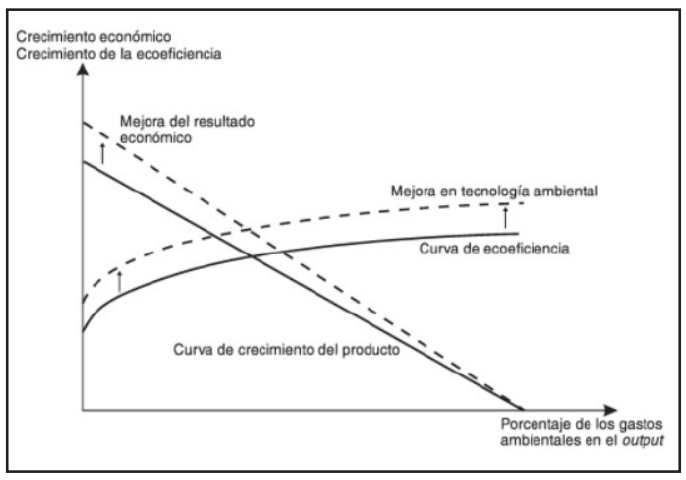

Fuente: (European Communities, 2002)

En general, los gastos ambientales para la empresa, al menos a corto plazo, representan un costo que inicialmente no proporciona un output con valor de mercado, a medio plazo, en cambio, es posible que refuercen su posición competitiva y la imagen de la empresa.

Desde el punto de vista de las empresas eléctricas en Costa Rica, se parte de la hipótesis que la curva de la mejora en tecnología ambiental, al utilizar modelos de smart grid, es proporcional a la mejora en la curva de eco-eficiencia, lo cual se comprueba con el crecimiento económico, al obtener una disminución del porcentaje de gastos u outputs en contra del ambiente.

De la definición de eco-eficiencia se conoce que el porcentaje de crecimiento de la presión ambiental $(\Delta E / E)$ es igual al porcentaje de crecimiento $(\Delta Y / Y)$ del output menos la mejora en eco-eficiencia $(\Delta P / P)$, matemáticamente, según la siguiente ecuación (European Communities, 2002):

$$
\frac{\Delta \mathrm{E}}{\mathrm{E}}=\frac{\Delta \mathrm{Y}}{\mathrm{Y}}-\frac{\Delta \mathrm{P}}{\mathrm{P}}
$$

La teoría ofrece un efecto de mejora en los resultados económicos, esto por cuanto las empresas eléctricas están bajo el efecto regulatorio, por tanto, parte de la disociación económica está directamente relacionado a las tarifas.

El modelo a proponer ofrecería la opción de suponer que el gobierno puede incidir en el nivel de gasto ambiental de las empresas eléctricas en Costa Rica, por medio de cambios en la regulación, por ejemplo, que parte de la tarifa sea dedicada a la inversión de nuevas tecnologías, como lo es la eco-eficiencia, por medio de la propuesta de utilización de smart grid.

El caso supone que, la preferencia sobre la regulación se representa a través de las curvas de indiferencia, que simbolizan niveles de utilidad superiores ante presiones ambientales menores y mayores niveles del output, por lo tanto, la regulación para las empresas eléctricas debe optimizar las decisiones eligiendo el nivel de gasto ambiental que le sitúa en la curva de indiferencia de mayor nivel de utilidad.

Por tanto, las mejoras en tecnología ambiental, evidentemente conducen a un incremento en la tendencia de la tasa de mejora en eco-eficiencia $\pi$, reduciendo la tasa de crecimiento de la presión ambiental para cada nivel de gasto ambiental (European Communities, 2002).

\section{Metodología}

En la econometría existen diversas escuelas de pensamiento sobre la metodología, pero la que más se utiliza es la tradicional o clásica, la cual aún predomina en la investigación económica y las ciencias sociales. Esta metodología se ajusta a los siguientes lineamientos (Guajarati \& Porter, 2010):

1. Planteamiento de la teoría o de la hipótesis.

2. Especificación del modelo matemático de la teoría.

3. Especificación del modelo econométrico o estadístico de la teoría.

4. Obtención de datos.

5. Estimación de los parámetros del modelo econométrico.

6. Pruebas de hipótesis.

7. Pronóstico o predicción.

8. Utilización del modelo para fines de control o de políticas.

Este artículo pretende realizar un acercamiento de un modelado econométrico, en donde se exponen primero los lineamientos de un planteamiento de la teoría económica, luego la especificación del modelo matemático de dicha teoría y finalmente la aproximación de la especificación del modelo econométrico, enfocado en que las empresas eléctricas en Costa Rica puedan determinar si al aplicar tecnología como la de un smart grid, pueden contribuir con el desarrollo sostenible, la competitividad en la industria, coadyuvar con la matriz energética nacional, reducir tarifas y obtener crecimiento y beneficios económicos (Guajarati \& Porter, 2010).

\section{Planteamiento de la teoría o de la hipótesis.}

En el planteamiento de la teoría, se parte de una economía reconocida, en otras palabras, la econometría tiene que ver con la determinación empírica de las leyes económicas. 
Este artículo realiza el trazado de la hipótesis en la ecuación del crecimiento económico de Harrod-Domar, en función del ahorro y la inversión.

Una vez establecida la ley económica en la cual se basa el modelo, se plantea la unificación con la eco-eficiencia, que establece la tasa de ahorro respecto a la renta real y se obtiene el modelo Harrod-Domar modificado del crecimiento en el output real.

Por lo tanto, se bosquejan interrogantes sobre qué ocurre con las mejoras en tecnología ambiental en las empresas eléctricas de Costa Rica, en donde supondría que al incrementar la inversión en temas ambientales y tecnológicos, puedan tener un ahorro en sus cuentas y por ende se pueda tener una mejor eficiencia y eficacia en sus operaciones, llevando a que se pueda realizar una disminución en las tarifas eléctricas, que convendría tanto a nivel regulatorio, de competitividad empresarial y por ende a nivel país, y evidentemente conducen a un incremento en la tendencia de la tasa de mejora en eco-eficiencia $\pi$, reduciendo la tasa de crecimiento de la presión ambiental para cada nivel de gasto ambiental.

A consecuencia de la mejora en $\pi$, la curva de intercambio pivotearía hacia arriba, en la dirección de mejoras ambientales permitiendo, a su vez, un mayor crecimiento económico, como se mostró en la Gráfica 1.

Por lo tanto, se esboza la siguiente pregunta de investigación: ¿existe la capacidad económica a nivel de las empresas del sector eléctrico de Costa Rica, para la integración de redes inteligentes?, esta capacidad, ¿se podría calcular y llevar a la implementación de eco-eficiencia por medio de la implementación del smart grid y por ende al crecimiento económico?

Para ello, como se ha mencionado, se debe plantear un modelo matemático basado en la teoría económica de crecimiento de Harrod y Domar, modificado a un modelo econométrico como cualquier otro modelo de regresión lineal, en el sentido de que los parámetros $\beta 1$ y $\beta 2$ son lineales.

La diferencia es que la variable dependiente es el logaritmo de Y y la regresora o variable explicativa es el tiempo, que adquiere valores enteros desde 1 (Guajarati \& Porter, 2010).

Los modelos a aplicar se denominan semilog, porque solo una variable aparece en forma logarítmica y para fines descriptivos, un modelo en el cual la variable regresada es logarítmica; se denomina modelo log-lin. En el presente modelo, el coeficiente de la pendiente mide el cambio proporcional constante o relativo en $\mathrm{Y}$ para un cambio absoluto, dado en el valor de la regresora, por lo que se tiene la ecuación (Guajarati \& Porter, 2010).

$$
\beta_{2}=\frac{\text { cambio relativo en regresada }}{\text { cambio absoluto en la regresora }}
$$

Si se realiza la multiplicación del cambio relativo en $\mathrm{Y}$ por 100 , de la fórmula anterior, dará entonces el cambio porcentual, o la tasa de crecimiento, en $Y$ ocasionada por un cambio absoluto en $X$, la variable regresora. Es decir, 100 por $\beta 2$ da como resultado la tasa de crecimiento en Y; 100 por $\beta 2$ se conoce como la semi-elasticidad de Y respecto de $X$ (Guajarati \& Porter, 2010).

Desarrollar la especificación del modelo matemático de la teoría, es el paso siguiente en este artículo, como lo muestra la metodología en su punto número 2 , y se generan las siguientes interrogantes, ¿qué variables dependientes e independientes a nivel de tendencias tecnológicas se deben de estudiar en las empresas eléctricas en Costa Rica, para determinar el modelo econométrico?

Por lo que a partir de la siguiente ecuación 25, se desarrollará el modelo econométrico que demuestre cuantitativamente si las empresas eléctricas están en capacidad de invertir en tecnología que las vuelva eco-eficientes y demuestren crecimiento.

$$
\frac{\Delta \mathrm{E}}{\mathrm{E}}=\frac{\Delta \mathrm{Y}}{\mathrm{Y}}-\frac{\Delta \mathrm{P}}{\mathrm{P}}
$$

\section{Análisis y Discusión}

Como se ha mencionado en la introducción, el artículo plantea la especificación del modelo econométrico o estadístico de la teoría, mostrado en la ecuación 25, para desarrollar en una futura tesis doctoral, los siguientes pasos de la metodología económica; Especificación del modelo econométrico o estadístico de la teoría, Obtención de datos, Estimación de los parámetros del modelo econométrico, Pruebas de hipótesis y Pronóstico o predicción.

\section{Datos y aproximación empírica}

Para el análisis y discusión, se plantea de la metodología económica, que las variables de un modelo empírico, es una representación del verdadero proceso de generación de datos, y su finalidad es mostrar una serie de variables en las cuales se puede desarrollar una tesis, donde se haga el modelado econométrico, con todas las aristas económicas de tecnología, las diferentes recombinaciones paramétricas, y otras en las cuales se pueda firmar la hipótesis planteada.

Por ejemplo, un modelo empírico es teóricamente consistente si reproduce el modelo teórico en las condiciones supuestas por la teoría. Por otro lado, el modelo empírico se admite si sus predicciones satisfacen todas las restricciones de los datos con probabilidad (Hendry \& Richard, 1982). 
Para sustentar el modelo empírico, las empresas eléctricas del país, deben delimitar nuevos estándares o normalizaciones, así como nuevas opciones de gestión estratégica, en donde se debe unir la disyuntiva entre la administración de las empresas eléctricas y las áreas técnicas operativas, temas analizados en un estudio que realizó el MIT Massachusetts Institute of Technology denominado The Future of the Electric Grid, and Interdisciplinary MIT Study, elaborado en el año 2011 (Massachusetts Institute of Technology, 2011).

Este informe, el quinto de una iniciativa denominada "Future of", serie emitida por el área de Energía del MIT, indica la necesidad de integrar la red eléctrica en este caso de EE.UU. confirmando con este reporte, la visión a nivel global de la identificación y análisis de las áreas políticas en cuando al establecimiento de la red inteligente o smart grid (Massachusetts Institute of Technology, 2011).

Las nuevas tendencias a nivel de países en vías de desarrollo, han mostrado nuevos modelos de cara a una empresa eléctrica inteligente y han incluido una metodología denominada "modelo de madurez para el smart grid", que es la tendencia más reciente para las empresas eléctricas. El Modelo de Madurez de un smart grid, es una herramienta de gestión bajo la dirección del Instituto de Ingeniería de Software de la Universidad Carnegie Mellon. El modelo fue desarrollado originalmente para el uso de los servicios públicos de energía eléctrica (Smart Grid, 2011).

El modelo proporciona un marco para entender el estado actual de la implementación de redes inteligentes y su capacidad dentro de una empresa eléctrica, además proporciona un contexto para el establecimiento de futuras estrategias y planes de trabajo, ya que pertenecen a un crecimiento inteligente con implementaciones del tipo cuadrícula. Está compuesto por ocho dominios que contienen cada uno seis niveles definidos de madurez, que van desde el Nivel 0 (más bajo) al nivel 5 (más alto) (Smart Grid, 2011).

Estos niveles establecen un marco para determinar el estado actual del desarrollo y capacidad de redes inteligentes en una empresa del sector eléctrico, también establece un contexto para determinar estrategias de futuro y planes de trabajo enfocados hacia la tecnología y las redes inteligentes.

Los dominios son agrupaciones lógicas de capacidades y características relacionadas con las redes inteligentes, en donde las empresas determinan basadas en un cuestionario, cuál es el nivel que se encuentran. La idea es desarrollar una fórmula económica que establezca si los costos de inversión en las estrategias del modelo de madurez y más aún en la tecnología, obedece a la demostración teórica de que existe crecimiento y con ello las empresas eléctricas puedan tomar la decisión de realizar la inversión que esto conlleva.
Los niveles del modelo de madurez son los siguientes (Smart Grid, 2011):

- $\quad$ Estrategia, Gestión y Regulación(SMR): Desarrollo de visión $\mathrm{y}$ estrategia hacia red inteligente, desarrollo de procesos de gestión, promoción de relaciones con patrocinadores.

- Organización y Estructura (OS): Cambios en cultura, estructura, comunicaciones, recursos humanos y gestión del conocimiento.

- Operaciones de red (GO): Operación confiable, segura, con suministro a largo plazo y eficiente. Operaciones automatizadas con flexibilidad.

- Gestión de activos y trabajo (WAM): Gestión óptima de los activos y recursos de trabajo. Uso efectivo de la cantidad de información disponible.

- Tecnología (TECH): Planeación estratégica para funcionalidades de red inteligente. Establecimiento de procesos de ingeniería y negocio para la evaluación, adquisición, integración y pruebas para nuevas tecnologías.

- Consumidor (CUST): Participación del consumidor: Pasiva o Activa.

- Integración Cadena de Valor (VCI): Habilidad para lograr objetivos administrando las interdependencias organizacionales en la cadena de suministro y de demanda, usando un suministro dinámico y gestión de la demanda basado en información en tiempo real.

- $\quad$ Sociedad y Ambiente (SE): Objetivos sociales con respecto a confiabilidad, seguridad y suministro de la infraestructura eléctrica, cantidad y fuentes de energía. Impacto de la infraestructura y la energía en el ambiente y la calidad de vida.

Los niveles de madurez representan estados definidos del progreso de una organización hacia logros en materia de redes inteligentes, tales como (Smart Grid, 2011):

- Automatización.

- Eficiencia.

- Confiabilidad.

- Ahorro de energía y costos.

- Integración de fuentes alternativas.

- Interacción con el consumidor.

- Acceso a nuevas oportunidades de negocio y mercado.

Basado en lo anterior, por ejemplo, en una de las principales empresas eléctricas en Costa Rica se desarrolló la aplicación del modelo de madurez de smart grid, lo que permitió brindar una aproximación de la condición inicial, llamada por algunos "base", del nivel de aplicación de las características esperadas para cada dominio en una empresa de distribución de energía eléctrica. 
El mismo fue aplicado en el 2015, iniciando con una inducción de lo que se realiza en la empresa, seguido de la aplicación del modelo de madurez, que proporcionó como resultado un "Nivel Por Defecto" en los diferentes dominios, que se muestran en el siguiente Gráfico 2.

\section{Gráfico 2. Diagnóstico SGMM}

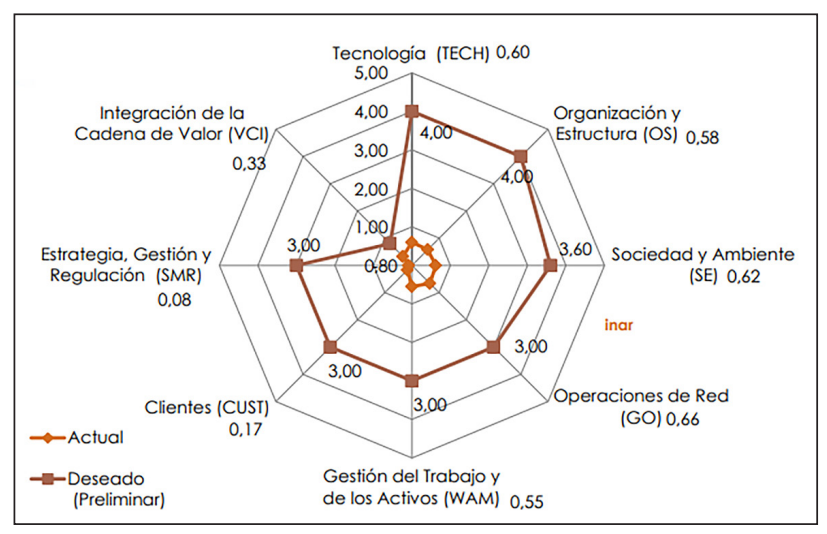

Fuente: Gerencia, 2015

Basado en el resultado del nivel de la empresa, se deben ejecutar acciones para poder escalar en los siguientes niveles, hasta llegar a la implementación del smart grid. Así por ejemplo en el tema tecnológico, en el cual se pretende demostrar el crecimiento de la empresa, se encuentra en el nivel 1, ya que únicamente llegó a obtener 0,6 de un esperado de 4 en la escala determinada, lo que obliga a tomar medidas como por ejemplo (Massachusetts Institute of Technology, 2011):

- Evaluar formalmente la arquitectura Tecnología de la - Información TI propuesta o existente con los atributos de calidad.

- Identificar en toda la empresa donde la tecnología podría mejorar el rendimiento de departamentos funcionales.

- Establecer un proceso formal en todas o la mayoría de las líneas de negocio para evaluar y seleccionar tecnologías armonizado con la visión/estrategia.

- Armonizar las inversiones claves en TI con la arquitectura TI empresarial en todas las líneas de negocio.

- Seleccionar las normas estándares que apoyen el desarrollo de su estrategia smart grid dentro de su arquitectura TI empresarial.

Ahora bien, para poder reproducir el modelo teórico que demuestre cuantitativamente si las empresas eléctricas del país están en capacidad de invertir en tecnología que las vuelva eco-eficientes y demuestren crecimiento, se basará en las predicciones del modelo econométrico de tecnología utilizando el fundamento del smart grid, la idea es cuantificar las acciones resultantes del diagnóstico del modelo de madurez, y establecer: criterios de costos, inversiones, uso de tecnología, evaluación de lo utilizado, capital actual, modelo tarifario, planillas, demanda del cliente, demanda energética, modelos de eco-eficiencia, y otras variables que se deben de adherir a la estructura básica que se pretende realizar del modelado econométrico basado en la ecuación 24, y econométricamente en la teoría de semilog de la ecuación 25 .

Para ilustrar una aproximación empírica, utilizando tecnologías del modelo de madurez del smart grid, en el presente artículo se seleccionaron tres modelos econométricos ya desarrollados a nivel mundial, indicando que, el modelado econométrico por proponer es expandible y, estas analizan las principales fuerzas motrices e interacciones dentro del sistema eléctrico para cuantificar: el impacto en la carga máxima, tecnologías de energía limpias, redes inteligentes y apoyo a las políticas regulatorias (Heinen, Elzinga, Seul-Ki , \& Ikeda, 2011).

Los tres modelos seleccionados como ejemplo son: medidores inteligentes, el número de vehículos eléctricos y la respuesta a la demanda, los tres con desarrollos propios de smart grid (Heinen, Elzinga, Seul-Ki , \& Ikeda, 2011).

\section{Medidores Inteligentes}

Los medidores inteligentes o denominados AMI por Advanced Meter Infraestructure en inglés, es un equipo que permite la facturación dinámica de la electricidad midiendo el consumo en intervalos de hora o más pequeños. Este medidor también podría comunicarse con la pantalla en el hogar dentro de las instalaciones del cliente y mostrar el consumo en tiempo real y el precio de la electricidad.

La penetración de $A M I(\%)$ representa la proporción de usuarios finales conectados a la red eléctrica a través de medidores inteligentes. Los medidores inteligentes sin tarifas eléctricas dinámicas, sin embargo, no proporcionan ningún incentivo para gestionar el pico de la demanda (Heinen, Elzinga, Seul-Ki , \& Ikeda, 2011).

Con el fin de evaluar el impacto potencial en la demanda máxima, la cuota de los medidores inteligentes instalados se introduce como una variable auxiliar para medir el despliegue de AMI (Heinen, Elzinga, Seul-Ki , \& Ikeda, 2011).

Se puede suponer que la implementación de medidores inteligentes sigue la forma de una curva logarítmica con crecimiento exponencial inicialmente, seguida por un crecimiento lento y finalmente alcanzando la madurez, ya que la mayoría de los hogares estarían equipados con medidores inteligentes. Por lo tanto, el despliegue de AMI puede ser representado por las siguientes ecuaciones (Heinen, Elzinga, Seul-Ki , \& Ikeda, 2011): 


$$
\begin{gathered}
\frac{\mathrm{dAMI}}{\mathrm{dt}}=\mathrm{r} * \operatorname{STEP}\left(\mathrm{T}_{\mathrm{DR}}-\mathrm{T}_{\mathrm{Lag}}\right) * \\
\operatorname{AMI}(\mathrm{t}) *(1-\operatorname{AMI}(\mathrm{t}))
\end{gathered}
$$

El coeficiente $\mathrm{r}$ es la tasa de crecimiento. Se supone que la función de paso STEP se activa en un intervalo de tiempo fijo $\left(T_{L a g}\right)$ antes del momento en que la respuesta de demanda (TDR) está en funcionamiento, con $T_{\text {Lag }}$ asumido como cinco años. La función STEP en la discusión anterior se define como (Heinen, Elzinga, Seul-Ki \& \& Ikeda, 2011):

$$
\mathrm{STEP}=\left\{\begin{array}{l}
0 \mathrm{si} \mathrm{T}_{\mathrm{DR}}-\mathrm{T}_{\mathrm{Lag}}<0 \\
1 \mathrm{si} \mathrm{T}_{\mathrm{DR}}-\mathrm{T}_{\mathrm{Lag}}>0
\end{array}\right.
$$

\section{Demanda de Generación}

La demanda de generación $\left(D_{G E N}=\right.$ terawatthora $\left.(T W h)\right)$ indica el consumo de electricidad estimado por las proyecciones de la Perspectiva Tecnológica de Energía o ETP por sus siglas en inglés, éstos consideran mejoras en la eficiencia energética y que según la teoría no están influenciadas por las redes inteligentes desplegadas en este modelo (Heinen, Elzinga, Seul-Ki , \& Ikeda, 2011).

No se espera que los programas de respuesta a la demanda reduzcan la demanda, sino simplemente cambien el consumo del pico a la hora punta. Las redes inteligentes pueden reducir la demanda de generación reduciendo las pérdidas de transmisión y distribución a través de avanzadas herramientas de control y monitoreo.

Por lo tanto, los números de ETP se ajustan mediante un coeficiente de eficiencia $\alpha$ como sigue (Heinen, Elzinga, Seul-Ki , \& Ikeda, 2011):

$$
\mathrm{D}_{\mathrm{GEN}}=\propto(\mathrm{t}, \mathrm{AMI}) * \mathrm{D}_{\mathrm{GEN}}^{\mathrm{ETP}}(\mathrm{t})
$$

\section{Vehículos Eléctricos}

$\mathrm{Al}$ analizar los Vehículos Eléctricos $\left(\mathrm{EV}_{\mathrm{s}}\right)$ y los Vehículos Eléctricos Híbridos Enchufables $\left(\mathrm{PHEV}_{\mathrm{s}}\right)$, desde el punto de vista de las empresas eléctricas en Costa Rica, se debe considerar que son elementos alimentados por una batería eléctrica y que ésta a su vez debe ser cargada desde la red, denominada "grid-to-vehicle" (G2V). Si está conectado durante las horas punta y si el acceso a la red no se gestiona de manera apropiada, los vehículos aumentarán la carga máxima (Heinen, Elzinga, Seul-Ki , \& Ikeda, 2011).

Sin embargo, también tienen el potencial de actuar como dispositivos de almacenamiento de red y la alimentación de la electricidad de nuevo en la red en horas pico. Esta función se denomina vehículo a la red $(\mathrm{V} 2 \mathrm{G})$ y se basa en una red inteligente que permite una mayor participación del lado de la demanda para el equilibrio, la reducción de picos de las acciones de contingencia (Heinen, Elzinga, Seul-Ki , \& Ikeda, 2011).

Por lo tanto, el potencial real de almacenamiento disponible de EVs / PHEVs en este modelo depende de la implementación de la red inteligente, que a su vez está determinada por la proporción de EVs / PHEVs en combinación con medidores avanzados (AMI) y precios de tiempo de uso. La potencia que un vehículo puede entregar puede ser aproximada con la siguiente fórmula (Heinen, Elzinga, Seul-Ki , \& Ikeda, 2011):

$$
\mathrm{P}_{\mathrm{VEH}}=\frac{\left(\mathrm{E}_{\mathrm{s}}-\eta_{\frac{\mathrm{EV}}{\mathrm{PHEV}}} * \mathrm{~d}_{\mathrm{D}}+\mathrm{d}_{\mathrm{RB}}\right) * \eta_{\mathrm{INV}}}{\mathrm{t}_{\mathrm{DISP}}}
$$

Donde, $P_{V E H}$ es la potencia máxima de $\mathrm{V} 2 \mathrm{G}(\mathrm{kW}), E_{s}$ la energía almacenada disponible como corriente continua $(\mathrm{kWh})$ al inversor; $\mathrm{dD}$ la distancia recorrida $(\mathrm{km})$ ya que la batería estaba llena; $d_{R B}$ la distancia $(\mathrm{km})$ del buffer de rango requerido por el conductor; $P_{H E V}$ la eficiencia de conducción eléctrica del vehículo $(\mathrm{kWh} / \mathrm{km}) ; \eta_{I N V}$ la eficiencia de conversión eléctrica del inversor de corriente directa a alterna (sin dimensiones); y $t_{D I S P}$ el tiempo que la energía almacenada del vehículo es despachada (horas) (Heinen, Elzinga, Seul-Ki , \& Ikeda, 2011)

\section{Planteamiento de tesis}

Basados en los tres ejemplos anteriores, suponemos ahora un modelado econométrico hipotético, el cual sería desarrollado más ampliamente en la tesis doctoral, en este caso se presentan tres variables: medidores inteligentes, generación a la demanda y vehículos eléctricos, de las múltiples opciones que se deben de desarrollar en la investigación, basados en el modelo de madurez de smart grid presentado, para luego realizar la prueba de hipótesis de que las empresas al correr el modelo econométrico, su resultado indique si es factible económicamente tener crecimiento por los ahorros eco-eficientes.

Si se unifican por el momento, estas tres variables: infraestructura de medición avanzada, vehículos eléctricos y generación de la demanda, con el modelo econométrico y de eco-eficiencia de la fórmula 25 , se pude obtener un modelado inicial, en el cual se pude analizar de la siguiente manera:

$$
\frac{\Delta \mathrm{E}}{\mathrm{E}}=\frac{\Delta \mathrm{Y}}{\mathrm{Y}}-\left(\frac { \Delta \mathrm { P } } { \mathrm { P } } \left\{\left[\begin{array}{c}
\propto(\mathrm{t}, \mathrm{AMI}) * \mathrm{D}_{\mathrm{GEN}}^{\mathrm{ETP}}(\mathrm{t}) \\
\frac{\left(\mathrm{E}_{\mathrm{s}}-\eta_{\text {PHVV }} * \mathrm{~d}_{\mathrm{D}}+\mathrm{d}_{\mathrm{RB}}\right) * \eta_{\text {INV }}}{\mathrm{t}_{\mathrm{DISP}}} \\
\mathrm{r} * \operatorname{STEP}\left(\mathrm{T}_{\mathrm{DR}}-\mathrm{T}_{\mathrm{Lag}}\right) * \operatorname{AMI}(\mathrm{t}) *(1-\operatorname{AMI}(\mathrm{t}))
\end{array}\right)\right.\right.
$$


Por lo que tenemos; de la definición de eco-eficiencia, el porcentaje de crecimiento de la presión ambiental $(\Delta E / E)$ es igual al porcentaje de crecimiento $(\Delta Y / Y)$ del output menos la mejora en eco-eficiencia $(\Delta P / P)$, matemáticamente, se incluye una matriz con las variables antes vistas; suponiendo que al incluir lectura remota se tiene una mejora en el control de carga y por ende una disminución del consumo de la energía eléctrica, que viene a coadyuvar a la matriz de generación de energía eléctrica.

Por otro lado, al introducir vehículos eléctricos, los mismos se pueden considerar como almacenadores de energía y por ende baja las curvas de carga en las empresas eléctricas, reduciendo los picos de demanda y el uso de hidrocarburos en la generación de electricidad.

Como se mencionó, el modelado econométrico supone ser expandible, para ingresar más ecuaciones econométricas a la matriz planteada en la hipótesis fórmula 30, y así poder demostrar, finalmente la aproximación de la especificación del modelo econométrico enfocado en que las empresas eléctricas en Costa Rica puedan determinar, si al aplicar tecnología como la de un smart grid, pueden contribuir con el desarrollo sostenible, la competitividad en la industria, coadyuvar con la matriz energética nacional, reducir tarifas y obtener crecimiento y beneficios económicos, lo que supondría que sí se tiene mejora en la eco-eficiencia $(\Delta \mathrm{P} / \mathrm{P})$ $\mathrm{y}$ lograr que la presión ambiental disminuya y sea el factor crecimiento el que aumente.

\section{Conclusiones}

Se ha determinado que el modelo económico de HarrodDomar, es la base económica fundamental para establecer que el diferencial de ahorro es igual al diferencial de inversión, que asume que una organización, en este caso de estudio las empresas eléctricas en Costa Rica, pueden demostrar crecimiento, en el tanto puedan disminuir los costos de operación realizando inversiones en materia ambiental, bajo la premisa de la eco-eficiencia, esto porque se ha demostrado empíricamente que si las empresas invierten en tecnología de cara a los aspectos ambientales, como lo son el smart grid, pueden contribuir a ser más eficientes y efectivas, trasladando ese crecimiento en reducciones en costos operativos que vienen a contribuir con mejores tarifas, contribuir con la política energética nacional y además aportar a la competitividad de los sectores industrial y comercial.

Se mostraron ejemplos de modelados econométricos de tres de las variables dentro de una estructura de smart grid, las cuales obedecen a implementaciones de tecnologías que contribuyen con los aspectos ambientales, y que, desde el punto de vista de la eco-eficiencia, son propulsores en la disminución del porcentaje de gastos u outputs en contra del ambiente.

Estos inputs, son una parte de la tecnología que un modelo de madurez asume para poder integrar una red inteligente o smart grid, cada elemento como se puede observar tiene un modelo de análisis desde la perspectiva técnica y económica, las cuales en algunos casos ya han sido desarrolladas como los antes expuestos y otros deben de plasmarse en un modelo econométrico similar, para poderlos integrar a la fórmula número 30 , de eco-eficiencia básica que se plantea en este artículo.

La idea con este artículo, es incentivar el desarrollo de una futura tesis doctoral, cuyo objetivo sea la modelación econométrica de la eco-eficiencia en las empresas eléctricas de Costa Rica utilizando smart grid, con una sola matriz, por medio de la unión de los modelados económicos y econométricos de las variables tecnológicas que se deben de implementar.

Se debe ampliar en los casos que no se cuente con una fórmula econométrica, e investigar el aplicar en las variables tecnológicas en que sí se tengan fórmulas, así mismo cuantificar y adaptar un modelo econométrico global en una sola matriz, en la cual se obtengan datos que se deben de recolectar de las empresas eléctricas del país, los insumos para poder determinar si dichas empresas están en las condiciones económicas para poder implementar un smart grid, a su vez lograr demostrar que esas mejoras en tecnología logren disminuir las presiones ambientales y por ende lograr un crecimiento económico.

Lo deseable es, que al crecer las empresas eléctricas económicamente, se puede suplir la demanda de energía del país mediante una matriz energética óptima, promoviendo el uso eficiente de energía para mantener y mejorar la competitividad del país con predominio de fuentes renovables y al menor costo, con el cambio tecnológico, procesos de innovación, investigación y conocimiento de alto impacto como lo es una red inteligente y que se logre controlar completamente el consumo y generación de la energía, la calidad y diversidad de fuentes, siendo estas preferiblemente compatibles con el desarrollo sostenible del país, ya que abarca tanto a la empresa eléctrica como a la comunidad en donde se aplique.

Por último, queda plasmada la base económica para el desarrollo de la matriz econométrica, y por ende dar continuidad en un futuro trabajo de investigación para dar respuesta a la pregunta de investigación, si ¿existe la capacidad económica a nivel de las empresas del sector eléctrico de Costa Rica, para la integración de redes inteligentes?, y poder matemáticamente, económica y econométricamente demostrar si esta capacidad, podrá llevar a la implementación de eco-eficiencia por medio de la implementación del smart grid y por ende al crecimiento económico en las empresas eléctricas de Costa Rica. 
